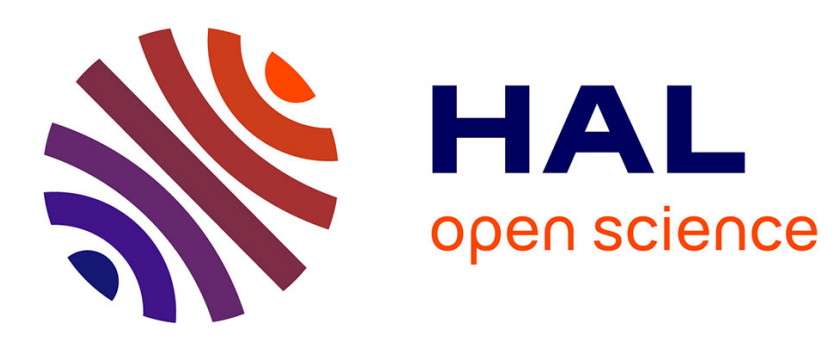

\title{
La plongée sous-marine, entre neutralisation du risque et affirmation de la sécurité
}

Gilles Raveneau

\section{To cite this version:}

Gilles Raveneau. La plongée sous-marine, entre neutralisation du risque et affirmation de la sécurité. Ethnologie française, 2006, Les sports à risques, 36 (4), pp.613. 10.3917/ethn.064.0613 . hal03135232

\section{HAL Id: hal-03135232 \\ https://hal.parisnanterre.fr/hal-03135232}

Submitted on 8 Feb 2021

HAL is a multi-disciplinary open access archive for the deposit and dissemination of scientific research documents, whether they are published or not. The documents may come from teaching and research institutions in France or abroad, or from public or private research centers.
L'archive ouverte pluridisciplinaire HAL, est destinée au dépôt et à la diffusion de documents scientifiques de niveau recherche, publiés ou non, émanant des établissements d'enseignement et de recherche français ou étrangers, des laboratoires publics ou privés. 


\section{La plongée sous-marine, entre neutralisation du risque et affirmation de la sécurité}

Gilles Raveneau

Université Paris X

\section{RÉSUMÉ}

La plongée sous-marine en milieu naturel est une activité qui modifie l'équilibre et la perception du corps. Les plongeurs - souvent convaincus de pratiquer une activité sans danger - s'exposent pourtant à des risques potentiellement mortels. À partir d'une enquête ethnographique, l'auteur montre qu'en définitive ce sport laisse une place aux transgressions des normes où le risque est progressivement converti en sécurité. Cet arrangement implique d'acquérir la maîtrise des "techniques de neutralisation " du risque, permettant aux plongeurs d'entretenir paradoxalement un système normatif, tout en le "violant ». Il apparaît ainsi que la transgression des normes de sécurité est moins un acte individuel qu'une conduite déterminée par l'organisation sociale de la plongée.

Mots-clés : Plongée sous-marine. Prise de risque. Norme. Transgression. Neutralisation.

Gilles Raveneau

Université Paris X

Maison de l'Archéologie et de l'Ethnologie

Laboratoire d'ethnologie et de sociologie comparative (UMR 7535 : CNRS-Paris X)

Laboratoire Sport et culture

21 , allée de l'Université

92023 Nanterre Cedex

gilles.raveneau@mae.u-paris10.fr

Contrairement à certains de mes travaux consacrés à la plongée "profonde " [Raveneau, 1999], l'angle d'analyse retenu dans le présent article vise à rendre compte du risque et de la prise de risque au plus près de cette activité, c'est-à-dire telle qu'elle se pratique majoritairement dans les clubs aujourd'hui. Loin de tout sensationnalisme ou de toute apologie des pratiques extrêmes et objectivement dangereuses, j'ai cherché à saisir la logique de la pratique ordinaire de la plongée en scaphandre. Je reconnais que tenir cet objectif ne fut pas facile, tant la clef de compréhension du risque et de sa justification semble résider dans les pratiques les plus extrêmes, sans parler de la fascination de l'ethnologue pour ces plongeurs fréquentant plus souvent que lui les grandes profondeurs.

Partant d'une recherche ethnographique «au long cours ${ }^{1}$, cet article se propose de traiter de la construction sociale d'une prise de risque ordinaire en plongée sous-marine. Mon hypothèse est que la pratique normalisée de la plongée fait une place au risque et à la prise de risque, tout en en effaçant les traces au fur et à mesure, de sorte qu'il s'agirait d'une activité où la règle consisterait dans la transgression même du système normatif. Ce qui va très exactement à l'encontre de la représentation dominante de l'activité chez les plongeurs eux-mêmes, et de ma propre représentation "indigène " de la plongée, en tant que plongeur sportif ordinaire. C'est dire le recul qu'il m'a fallu prendre pour mener à bien ce travail et ouvrir dans son prolongement de nouvelles perspectives fécondes sur la question.

À la charnière des deux représentations qui voudraient voir dans la plongée sous-marine une activité inoffensive ou au contraire une pratique dangereuse [Collard, 1998 ; Bonnet et al., 2003], je voudrais montrer que si le risque n'est pas étranger à la plongée sousmarine, ce n'est pas au sens où l'entendent généralement le spécialiste ou l'amateur lorsqu'ils parlent de sports. Le risque $^{2}$ sera considéré ici comme une forme de transgression légitimée à travers tout un processus de renormalisation. La valeur du risque serait ainsi recodée en sécurité et les plongeurs conduits à considérer certaines infractions comme normales, voire sécurisantes. Mais, avant d'en arriver là, il nous faut au préalable appréhender les modalités de l'expérience des plongeurs et saisir tout le concret des risques auxquels ils sont exposés. 


\section{- Les modalités de l'expérience}

Arrivé sur "site ", le plongeur, accompagné de coéquipiers, se prépare, s'habille et ajuste son scaphandre. Il vérifie le contenu de sa bouteille sous pression et le bon fonctionnement de son détendeur ${ }^{3}$. Lorsqu'il est prêt, il se "jette à l'eau " depuis le bateau ou le bord d'un rocher. En une ou deux minutes une fois immergé, le plongeur aura rejoint la profondeur voulue. Plus il descend et plus sa vitesse augmente. Peu à peu, il gonfle sa bouée pour ralentir sa descente et se stabiliser à la profondeur souhaitée. La lumière solaire pénètre de moins en moins et tout devient bleu. Les plongées en pleine eau s'effectuent sans repères ou presque. D'où la difficulté à s'orienter et l'intérêt de plonger sur des fonds rocheux. Le courant, les particules en suspension dans l'eau, la faible luminosité, l'absence de points de repère, tout cela participe d'une grande difficulté d'orientation et de la sensation de vertige, accentuée par les effets de la narcose ${ }^{4}$ à partir de 40 mètres. La descente dans le "bleu "s'effectue soit la tête la première, dans l'axe du tronc, ce qui constitue la position la plus hydrodynamique, avec rétablissement à l'approche du fond; soit debout, palmes en premier. La plongée sous-marine en milieu sauvage est une activité qui implique un bouleversement des données ordinaires de l'équilibre et de la perception du corps. On ne sera pas alors surpris d'apprendre que l'accomplissement dans cette pratique réside dans le contrôle de soi et de la situation par la valorisation du calme, de la quiétude, voire même de la lenteur.

Les modalités de l'expérience des plongeurs sont largement déterminées par les contraintes du milieu sousmarin, les gaz respirés et les lois physiques de la pression. Elles ont partie liée avec les conditions nécessaires au bon déroulement de l'activité. La plongée en milieu

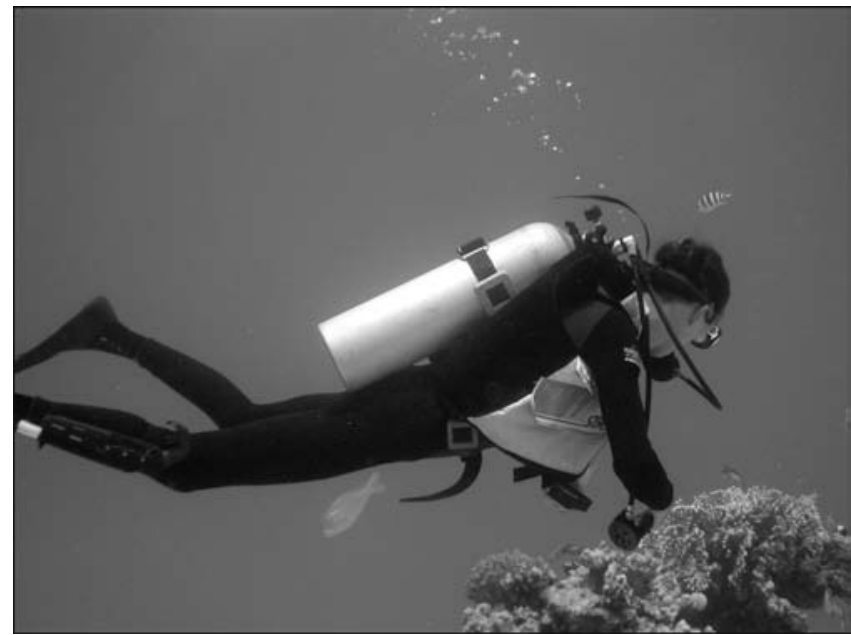

1. L'auteur lors d'une plongée d'exploration en mer Rouge, à Gamul Kibir (photo Roland Paillocher, 2004).

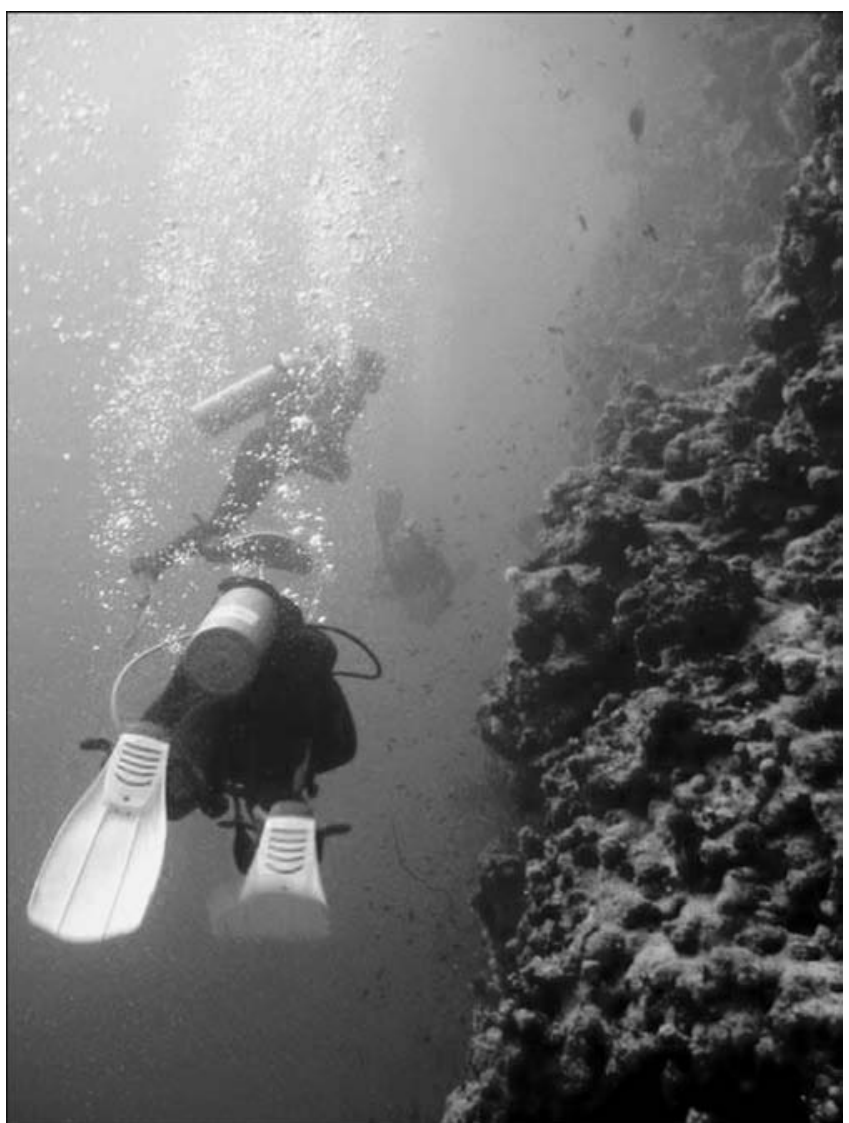

2. Déplacement le long d'un tombant en mer Rouge, à Dahab (photo de l'auteur, 1998).

naturel reste toujours incertaine ; elle est source de déséquilibre et d'incertitude, et ainsi inductrice de risques. La présence des risques ne peut donc pas être éradiquée, et ce sont même ces risques qui constituent le fondement de la pratique en milieu sauvage. Alors pourquoi les plongeurs interrogés persistent-ils à affirmer que leur activité est "sans risque " ? Et comment peuvent-ils en arriver à penser, si l'on en croit Luc Collard [op. cit. : 208], que la plongée est moins dangereuse que la gymnastique et le tennis?

Les plongeurs sont effectivement convaincus de l'innocuité de leur pratique. Pourtant, la réalité des risques encourus par les plongeurs est bien connue ${ }^{5}$. Mais elle a été comme évacuée par les réponses apportées par la formation reçue et les représentations partagées. Considérons par exemple l'un des manuels de référence dans le domaine, ayant servi à la formation de plusieurs générations de plongeurs, La plongée subaquatique de Philippe Molle et Pierre Rey ${ }^{6}$ : dès l'introduction, les auteurs notent qu' "il existe en effet un certain nombre de règles de sécurité que chaque plongeur doit connaître et respecter. La non-observation de ces règles provoque des accidents qui peuvent 
être graves, voire mortels. Loin de vouloir décourager le lecteur, nous espérons lui donner conscience très précisément des risques qu'il court [...] " [Molle et Rey, 1991: 7]. Si la nécessité de respecter les règles de sécurité est rappelée, les auteurs établissent aussi que l'activité expose à des risques, accidents ou mort, étant entendu que c'est le non-respect des règles de sécurité qui expose à ces risques. Il suffirait, à les lire, de s'en tenir au strict respect des normes de sécurité pour éviter les dangers. Or, je voudrais précisément montrer que la pratique de la plongée sousmarine porte au contraire à transgresser régulièrement le système normatif.

\section{- Prendre des risques}

Le sens commun commanderait que, parmi l'ensemble des individus également susceptibles d'enfreindre les règles de sécurité et de prendre des risques, seul un petit nombre d'entre eux le fassent. Mais est-ce si sûr? Et pourquoi ? J'ai avancé précédemment que la pratique de la plongée sous-marine expose à des risques et que ceux-ci font partie intégrante de l'activité, de sorte qu'il n'est pas possible de les éradiquer tous. Cependant, la pratique normale de la plongée, c'est-à-dire effectuée dans le cadre légal des règles de sécurité enseignées, réduit de fait les risques d'accident. Mais au-delà de ce risque normalisé, certains plongeurs dérogent parfois aux consignes de sécurité ou poussent plus loin leur engagement dans la pratique en prenant volontairement et consciemment des risques, certes mesurés et appréciés. Car il ne s'agit en aucun cas de formes dérivées de suicide ou de jeu de roulette russe, mais d'une décision rationnelle individuelle ou collective, soit avant la plongée, soit pendant l'immersion lorsque se présente telle ou telle opportunité (visiter une grotte sous-marine ou une épave, suivre un beau tombant au-delà de la profondeur fixée au départ, etc.). Écoutons Jasper (42 ans, enseignant, niveau $\mathrm{V}$ ) raconter comment il lui arrive de faire une "profonde" pour le plaisir : "Ce qui est certain, c'est qu'à un moment ou à un autre, je fais une sorte de fantaisie; mais je ne pense pas que ce soit un geste suicidaire, mais plutôt une volonté de casser le rythme. [...] On peut paraitre proche d'une prise de risque inutile, mais je crois que ce genre de conduite te vient des jours où tu te sens particulièrement bien. [...] À ce moment-là, pour le plaisir, je voulais descendre plus profond! Mais ça partait plus d'une positivité que d'une volonté suicidaire! Tu te sens bien à un moment donné, tu as envie de t'amuser! [...] Il fait beau, la mer est parfaitement calme, tout se passe comme sur des roulettes. Tout d'un coup, tu es sur un tombant, c'est très beau, ça descend, tu te laisses aller. C'est pas suicidaire, ni un défi pour un record! C'est, je suis bien, j'essaie! Je joue avec quelque chose! Ca te vient un beau matin, ça te prend sous l'eau! Ça te plaque presque au fond! C'est une fantaisie!"

Si la plongée " profonde" ou certaines performances comme celle de Jasper, capable de descendre à plus de 80 mètres de fond, sont assurément spectaculaires, il convient de souligner qu'une des formes les plus courantes et les plus pratiquées de ces prises de risque demeure la plongée ordinaire. Cela concerne par exemple les clubs associatifs où l'on pratique entre soi, avec ses propres guides de palanquée et moniteurs. Les directeurs de plongée règlent habituellement de manière autoritaire la composition des palanquées, ces formations associant des plongeurs censés évoluer ensemble. Leur objectif constant est d'équilibrer une palanquée en y plaçant comme chef d'équipe un plongeur plus aguerri et plus diplômé que les autres. Les critères de projets communs et d'affinités entre plongeurs passent après. Une infraction familière consiste d'ailleurs, après s'être concerté en surface pour le faire, à changer de coéquipier une fois sous l'eau. L'idée est simple : reformer une autre équipe sous l'eau, hors du contrôle de l'encadrement, pour que chaque nouvelle palanquée puisse réaliser son projet d'immersion. Défaire de la sorte l'association initiale pour la reformer avant la sortie de l'eau se fait en général sans difficulté. Mais il arrive que pour une raison ou pour une autre les plongeurs ne se retrouvent pas. L'arrangement est alors découvert ou pas, suivant la capacité d'observation et la duplicité des uns et des autres ; ainsi cette plongée au cours de laquelle était prévu un échange entre deux palanquées autonomes comprenant chacune deux plongeurs (l'une mixte, l'autre intégralement masculine), qui étaient convenues de se retrouver au mouillage. Les nouvelles palanquées formées après l'immersion se séparent: la nouvelle mixte fait ce qu'il est convenu d'appeler une "balade", c'est-à-dire une plongée d'exploration paisible à faible profondeur; l'autre projette une plongée plus engagée et plus profonde. Le parcours réalisé, l'air consommé (le seuil des 50 bars de sécurité dans la bouteille n'est toutefois pas atteint) et le temps écoulé (environ trente minutes) signent la fin de la plongée pour la palanquée mixte. Ils arrivent les premiers au mouillage, sous le bateau. Ils attendent l'autre équipe, qui ne vient pas, et voient défiler devant eux toutes les autres palanquées qui remontent. Ils se décident à faire surface à leur tour et rejoignent le bateau, se déséquipent et commencent à discuter intensément avec les uns et les autres de ce qu'ils ont vu. Les échanges se poursuivent sans que personne ne remarque l'absence des deux derniers plongeurs. On remonte l'ancre. Au bout d'un moment, dans le brouhaha des discussions et du rangement de l'équipement, le directeur de plongée, comme à son habitude, lance à la cantonade: "Tout le monde est là ?!", mais personne ne répond qu'il manque deux compagnons. Les deux plongeurs de la palanquée officieuse qui sont sur le bateau oublient jusqu'à l'existence de la palanquée officielle. Oui, chacun est bien remonté avec son coéquipier. Oui, c'est bien leur cas. Tout est normal donc. Le bateau part et rentre au port. Les deux plongeurs manquants sont en train de faire leur dernier palier de 


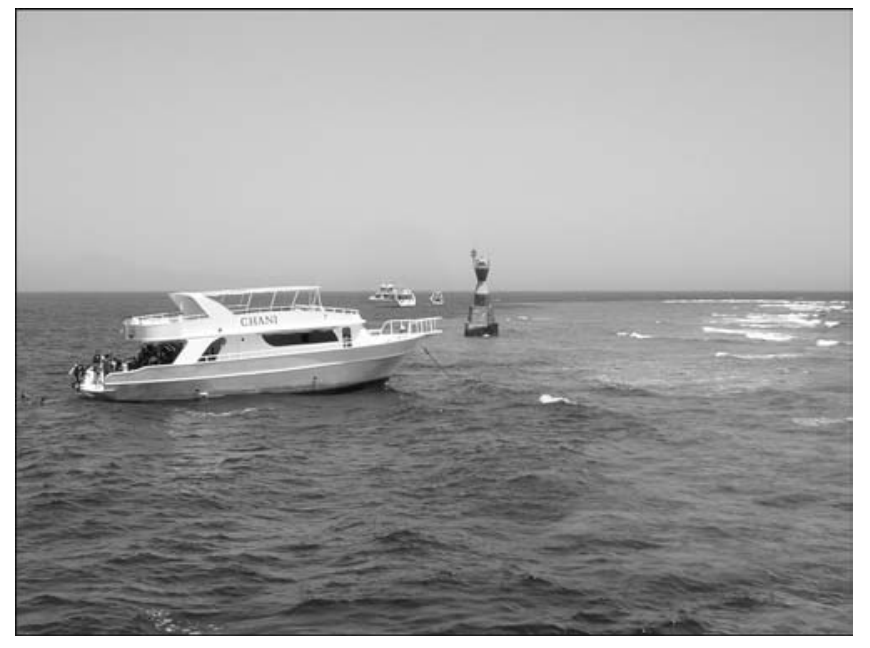

3. Bateau au mouillage, attendant les plongeurs en mer Rouge (photo de l'auteur, 2004).

décompression. Arrivée au port ; on décharge le bateau, et c'est à ce moment-là seulement qu'un des plongeurs se rend compte de l'absence de deux d'entre eux. Branle-bas de combat, discussion houleuse, fureur! Finalement, on apprend par radio qu'ils ont été récupérés par un autre bateau qui les ramène au port ${ }^{7}$.

Voici une autre situation de transgression et de prise de risque ordinaire: quatre plongeurs peu qualifiés (niveau II) décident de plonger ensemble à 45 mètres, alors qu'ils n'y sont aucunement habilités. En effet, il a été convenu avec le responsable du club et des palanquées qu'ils pouvaient plonger ensemble en respectant leur zone de prérogatives, c'est-à-dire jusqu'à 20 mètres de fond. Au-delà de cette profondeur, ils devaient être accompagnés d'un guide de palanquée ; ils le savaient. Ils descendent malgré tout à 45 mètres et «se font peur ». Deux sont narcosés, mais n'osent rien dire. L'intervention de l'un des plongeurs, qui en empoigne un et lui demande (par signes) si "tout va bien", est décisive. Il fait signe aux autres de remonter et évite sans doute ainsi l'accident. L'incident sera relaté, moqué et largement relativisé par la suite en petit comité, sans que jamais les responsables de plongée ne soient au courant. Ils font pourtant tous partie du même club et les rumeurs vont bon train. Ces expériences contribuent à créer des liens, des connivences et des affinités entre les plongeurs d'une association sportive, unissant certains et en excluant d'autres.

Autre cas : des plongeurs s'adressent, comme cela se fait beaucoup, directement à une structure commerciale pour effectuer leurs plongées. Le risque peut être alors un argument commercial: on vend de la sécurité, des sensations, des découvertes, du "risque " encadré en somme. Dans ce cas, le club fournit non seulement l'équipement, mais l'encadrement. Les contraintes de la plongée sont donc fixées par la structure d'accueil. Cependant, le niveau de pratique et les diplômes dont sont titulaires les plongeurs permettent différents cas de figure où les transgressions ne sont pas rares; comme celui typique de ces plongeurs autonomes que tout le monde attend parce que, ayant été trop profond par rapport aux consignes (horaires de remontée, profondeur, etc.), ils terminent leur palier de décompression au pendeur.

Toutes ces prises de risque n'engagent pas les pratiquants de façon identique, certaines étant potentiellement plus dangereuses que d'autres ${ }^{8}$. Toutefois, il est nécessaire de les apprécier en fonction du niveau de pratique et de maîtrise de l'activité, et de les pondérer en conséquence. Descendre à 45 mètres pour un plongeur de niveau III ou IV est une expérience banale, mais pour un plongeur de niveau I ou II il s'agit là assurément d'une transgression, dans la mesure où son diplôme de plongée et le type d'apprentissage suivi ne l'autorisent à le faire qu'à la profondeur maximale de 20 mètres. Pour être comparée à une autre, chaque prise de risque nécessite d'être replacée très exactement dans son contexte de réalisation (conditions du milieu, paramètres de la plongée, présence d'autres plongeurs qualifiés). L'appréciation objective comme la valeur subjective de ces prises de risque en dépendent. Mais là n'est pas encore l'essentiel. La prise de risque, quelle qu'elle soit, pose un problème théorique majeur : comment un pratiquant peut-il transgresser des normes et des règles de sécurité dont il admet pourtant la validité ?

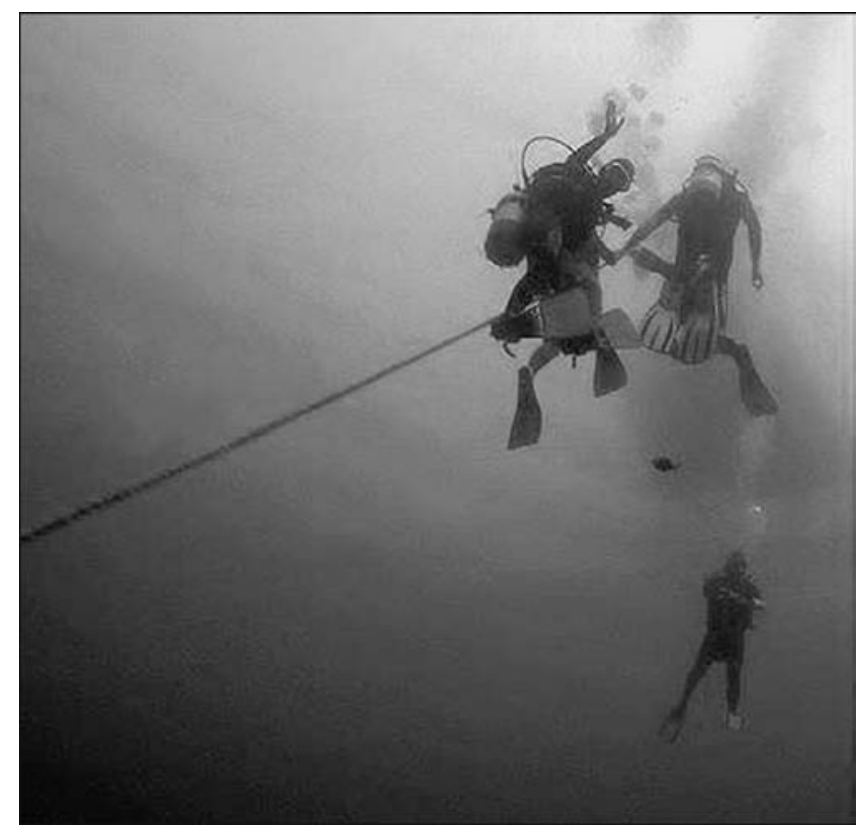

4. Fin de plongée et remontée le long de l'ancre sous le bateau, en Corse (photo de l'auteur, 1995). 


\section{- Des mesures de sécurité aux prises de risque, et retour}

Répondre à cette question peut se faire en deux temps : montrer d'abord qu'un consensus social s'est établi autour du risque, visant à banaliser et à contrôler ce dernier en l'intégrant directement dans les conditions d'apprentissage de la plongée, et en le recodant en sécurité ; établir ensuite que les plongeurs qui prennent volontairement des risques le font sans remettre en cause la validité des valeurs et des normes de sécurité de la plongée.

En partant de l'idée selon laquelle la prise de risque serait un moteur nécessaire à l'action et un facteur de progression, on renverse les perspectives habituelles qui font de l'interprétation du risque une positivité indésirable [Peretti-Watel, 2000], une ordalie [Le Breton, 1991, 2002], un calcul rationnel [Collard, op. cit.] ou une recherche de distinction sociale [Pociello, 1995]. Dans cette perspective, la prise de risque serait inscrite de manière légitime dans le code de la pratique, à travers notamment les différents exercices et expériences menés dans le cadre de la formation, mais recodée aussi symboliquement que concrètement en mesures de sécurité. Les diplômes de plongée, validés par des examens à la fois théoriques et pratiques, ne font pas autre chose. Ils soumettent le pratiquant à des situations de plus en plus périlleuses, tout en lui assurant l'apprentissage des techniques adaptées à ses capacités et l'acquisition des connaissances théoriques lui permettant de se mouvoir en immersion dans des conditions variées. On m'opposera ici que tout cela se fait dans des conditions de sécurité optimales et que nulle place n'est laissée à une prise de risque. Néanmoins, le novice est appelé à aller plus loin chaque fois, à dépasser sa peur et ses limites, à améliorer ses capacités physiques et sa connaissance du milieu. Il apprend à avoir confiance en lui et dans les autres en immersion, il comprend au fur et à mesure qu'il peut compter sur ses propres ressources et celles des autres membres de la palanquée pour faire face à l'imprévu. Certes, tout cela se fait progressivement, dans des conditions de surveillance et d'encadrement qui produisent plus de sécurité que de risque. Mais le plongeur débutant, comme du reste celui qui tend à mâtriser la technique, doit pourtant bien à chaque fois dépasser ses appréhensions, vaincre sa peur, modifier ses réactions et ses émotions, transformer ses performances motrices et sa consommation d'air, reconnaître le début d'un essoufflement ou d'une narcose, etc.

Dans le même mouvement, il apprend à reconnaître des sensations, à les nommer. En immersion, il acquiert ainsi des savoir-faire mentaux et corporels particuliers, un code de communication spécifique ${ }^{9}$; en surface, un langage et un système de valeurs propres à fournir des outils d'appréciation des opérations de plongée. Il est conduit à interpréter et à justifier ses actes, ses manœuvres et ses

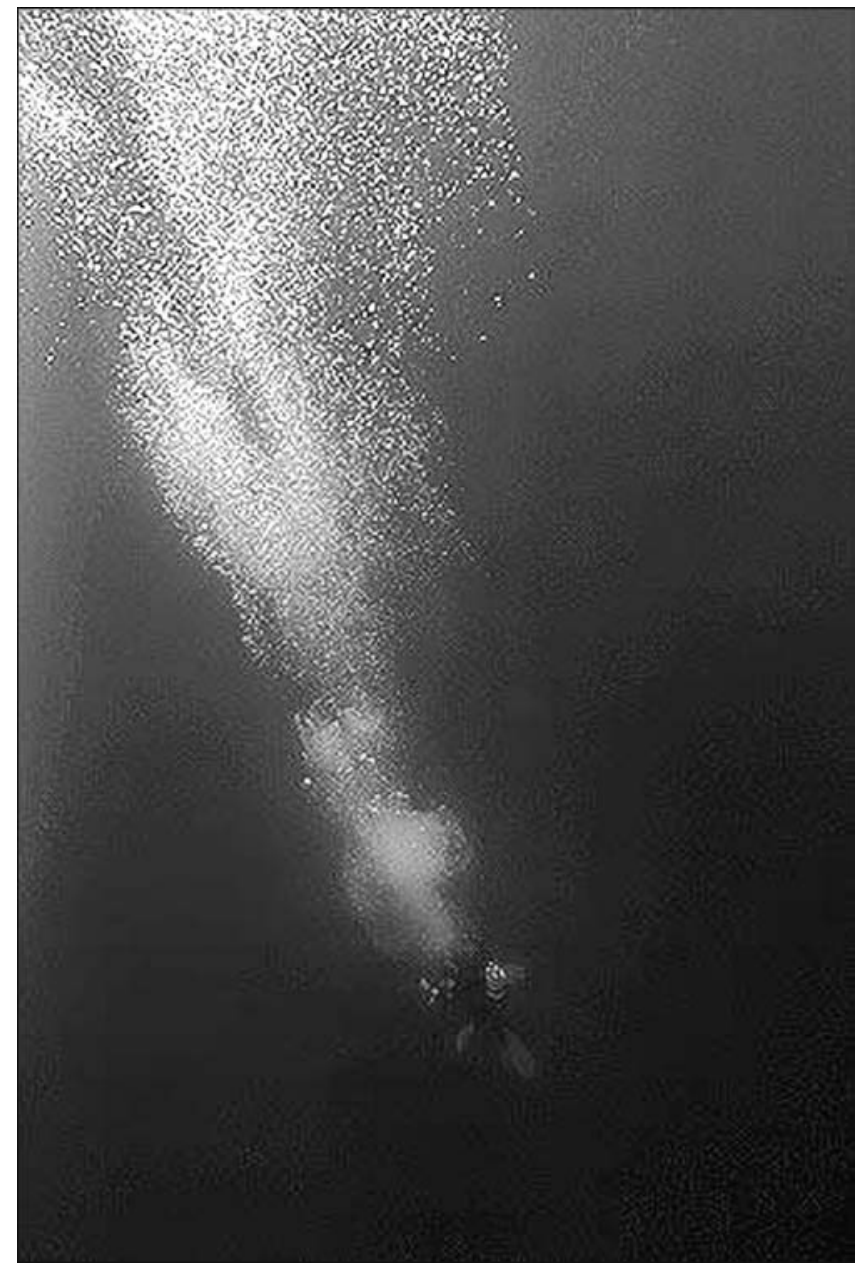

5. Remontée du fond, en Sardaigne (photo de l'auteur, 1996).

perceptions afin de leur donner une signification acceptable au regard du dispositif normatif. La prise de conscience des situations, des sensations, des représentations, des risques et de la sécurité est moins le résultat d'une compréhension individuelle que d'une appréhension collective. L'expérience pratique de la plongée, tout à la fois technique, sensorielle et langagière, est une action partagée, et ce, d'autant plus qu'il est théoriquement interdit de plonger seul. Une des règles de base veut justement qu'une immersion se fasse toujours au minimum à deux.

Ce que je veux souligner ici, c'est que l'apprentissage et la pratique normalisée de la plongée proposent, certes sous une forme atténuée et très contrôlée, des prises de risque nécessaires à la progression puis à la maîtrise reconnue de l'activité, une fois celle-ci acquise. D'où la présence banale du risque, recodé en sécurité, et le processus d'invisibilisation dont il est l'objet. On m'objectera sans doute qu'une fois atteints la compétence et le 
niveau requis, ce qui était jusque-là prise de risque ne l'est plus, disparaissant avec l'acquisition des moyens qui permettent d'y faire face. Mais c'est là bien vite oublier, d'une part, que les conditions ne sont jamais les mêmes ${ }^{10}$, d'autre part que la maîtrise de l'activité n'est jamais acquise une fois pour toutes. Elle nécessite d'abord un entraînement continu pour se maintenir à un haut niveau; ensuite, de nouvelles expériences pour étendre son champ d'action et se fixer ainsi de nouveaux objectifs, propres à maintenir le plaisir ressenti à l'exercice de l'activité. Ce que vise en dernière analyse l'apprentissage, c'est l'autonomie du plongeur, c'està-dire sa capacité à gérer seul, sans aide extérieure, sa plongée. Or, l'autonomie, c'est l'aptitude d'un individu à décider et à choisir sans avoir à en rendre compte à une instance supérieure. C'est la capacité de se donner des règles auxquelles on accepte de s'assujettir. On s'y soumet donc librement, et du fait même qu'elles existent ces règles permettent de s'accorder avec les autres. C'est en ce sens que l'« autonomie " en plongée est d'une part "réglée " par la logique interne de l'activité, de l'autre structurée par l'organisation sociale des plongeurs. C'est dire si les plongeurs "autonomes " (à partir du niveau III ${ }^{11}$ partagent une même conception de l'activité et sont aptes à décider de la conduite à tenir et des moyens, qu'ils soient techniques ou langagiers, de justifier leurs actions.

\section{- La banalité de la transgression}

Quels que soient leur sexe, leur âge ou leur profession, les plongeurs tiennent des propos très similaires sur l'approche qu'ils ont de la pratique. Ils envisagent la plongée comme une activité de découverte et de loisir dépaysante, engageant d'abord la maitrise de soi et de son autonomie à travers le contrôle du vertige et de la situation d'immersion, que viendrait renforcer une logique de calme et de la moindre dépense. Ils la considèrent ensuite comme une interaction avec des coéquipiers auxquels il convient d'être attentif, nécessitant de prévoir leur comportement et d'être soi-même prévisible. Cette interaction et cette surveillance mutuelle sont le gage d'une plus grande sécurité en cas de problème ou d'accident. Elles s'expliquent par l'aide qu'un plongeur peut apporter à un coéquipier en difficulté : essoufflement, narcose, perte de connaissance, manque d'air, blocage dans un filet ou un rocher, etc. Le plongeur se sait ainsi lié aux autres ; mais, dans la mesure où son immersion ne dépend plus seulement de lui, il doit pouvoir en retour anticiper les actions des coéquipiers impliqués dans la même situation. Plonger revient de la sorte à interpréter non seulement un milieu mouvant et incertain, mais aussi les comportements des autres. Un «bon plongeur " est alors celui qui sait anticiper chaque situation, et non appliquer sans discernement des règles générales. Ainsi pour ce plongeur interrogé : "Les règles de sécurité sont indispensables, tout ce que t'as appris, tout ça est utile, ce serait débile d'affirmer le contraire, mais à condition que ce soit adapté. [...] C'est comme la culture tu sais, c'est ce qui reste quand t'as tout oublié " (Pascal, 48 ans, enseignant, niveau IV). Par conséquent, les plongeurs doivent adapter leur conduite aux situations. Car si le strict respect de la règle convient parfaitement aux situations d'enseignement et d'examen, il peut aussi, dans d'autres circonstances, s'avérer dangereux. Les plongeurs autonomes estimeront donc normal et sans danger de transgresser certaines règles communes pour les adapter à la diversité des milieux et des situations. Trop différentes les unes des autres pour être comparables entre elles, celles-ci exigent en effet une adaptation de tous les instants au danger.

"Tu as des plongeurs, témoigne cet autre adepte, ils sont bons, bons pédagogues, bons théoriciens, ils savent te calculer des successives plus vite que leur ombre ou te faire un cours sur les accidents barotraumatiques. Ils sont bardés de diplômes, ils te diraient bientôt ce que tu dois faire! [...] Mais ils ont pas le sens marin, ils ne sont pas fluides. Tant qu'ils ne sont pas confrontés à des trucs un peu chauds, à des plongées qui ne sortent pas des bouquins, tu vois, tout va bien. Mais dès qu'il faut aller voir un peu plus loin, y'a plus personne. [...] Ils savent pas s'adapter à la nouveauté. Et je vais te dire, ils la recherchent pas non plus, hein ? la nouveauté. Ces gars-là, tu vois, ils sont forts dans la réflexion, mais pas dans l'action. C'est pas des bons plongeurs pour moi, c'est pas des brêles, non, mais c'est pas des bons plongeurs " (Francis, 53 ans, artisan, niveau V). Qu'est-ce que le "sens marin", la "fluidité "? Est-ce que cela existe ? Ce qui est sûr, c'est que pour Francis, cela se manifeste - ou non - en immersion, dans l'action. Et si cette " fluidité » dans l'eau n'était justement que l'expression de cette capacité d'adaptation qui demande disponibilité d'esprit et inventivité, de cette maîtrise individuelle fondée sur la réactivité et la compréhension, et non sur le respect à la lettre des règles ? La sécurité dans l'eau reposerait dès lors davantage sur ce sens de l'improvisation que sur la conformité des comportements au système normatif. D'où la banalité de toutes ces petites violations et réinterprétations du code de sécurité et de comportement que nous avons relevées précédemment. De façon plus générale, les plongeurs autonomes semblent penser, un peu à la manière des automobilistes [Peretti-Watel, op. cit. ; Assailly, 2001], que le risque d'accident viendrait surtout de ceux qui suivent à la lettre ce " code " et qui sont par là même incapables de s'adapter aux situations nouvelles, d'anticiper correctement les comportements de leurs coéquipiers et, en retour, de se montrer euxmêmes prévisibles pour autrui.

Mais comment accéder à cette "fluidité " et à ce "sens marin" ? Précisément par le non-souci des règles, leur négligence, leur mise entre parenthèses, autrement dit par une "dé-préoccupation" à leur égard. Puisque les voies d'ordinaire suivies pour maîtriser l'environnement 


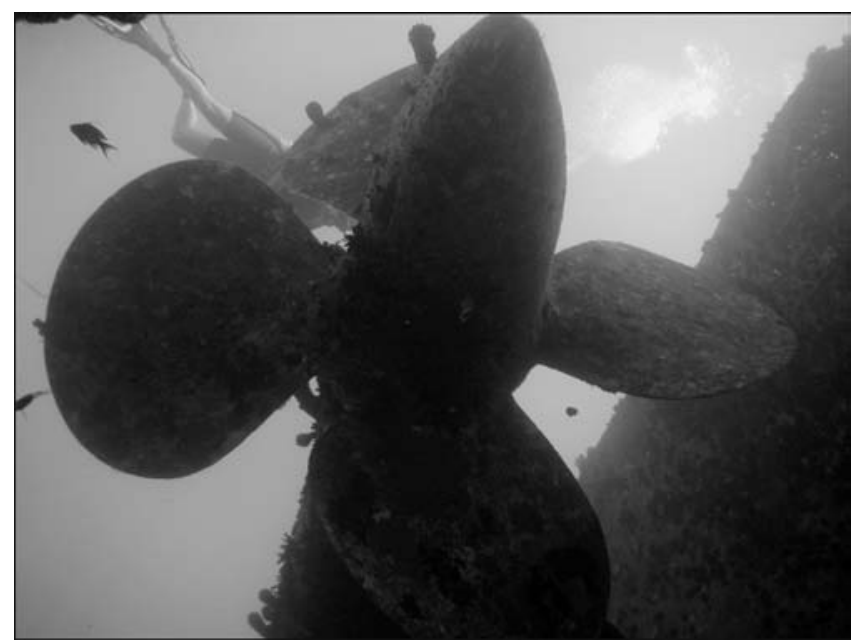

6. Visite d'une épave de bateau, le Salam Express, en mer Rouge. Vue sur l'hélice du navire (photo Frédéric Revert, 2004).

sous-marin et les situations rencontrées s'avèrent inefficaces et déplaisantes, la seule qui reste ouverte est celle de la transgression et du désapprentissage. La sociabilité et les interactions entre plongeurs autonomes conduiraient ainsi à faire de la transgression ordinaire une des règles du jeu de la pratique.

\section{- Flexibilité des règles de sécurité et techniques de neutralisation du risque}

Ces prises de risque (dans leur version contrôlée), nécessaires à la progression et à la reconnaissance de l'autonomie, font partie de la pratique banale de la plongée. L'ethnologue se méprendrait en considérant que les valeurs et les normes de conduite que l'activité sportive impose à ses adeptes opèrent à la manière d'impératifs catégoriques. Il conviendrait de les regarder plutôt comme "des guides qualifiés pour l'action, dont les recommandations sont limitées par le temps, l'espace, les personnes, et les circonstances sociales" [Sykes et Matza, $1957: 666$ ]. Ce qui signifie que le régime normatif de la plongée sous-marine admet une certaine flexibilité et que les règles de sécurité ne sont pas scrupuleusement respectées en toutes circonstances. Reconnaitre cette flexibilité, c'est accepter le fait qu'une transgression du système normatif puisse concerner ce dernier sans chercher pour autant à le remettre en cause, de sorte que le pratiquant qui enfreint les règles de sécurité dispose toujours de la possibilité de justifier ses actes afin de leur donner une signification acceptable. Autrement dit, le fautif est à même de produire un raisonnement montrant que les faits qui lui sont reprochés ne dérogeaient pas vraiment aux règles de sécurité. C'est ainsi qu'un plongeur peut à la fois transgresser des normes et en admettre la validité.

Or, s'il est possible de rationaliser une conduite après coup, rien n'interdit en principe de le faire avant et d'anticiper ainsi sur ce qu'on projette de faire, comme dans l'exemple de l'échange de partenaires de palanquée après l'immersion. À partir de là, il est possible de renverser complètement la perspective de la transgression, reconnue comme telle et à ce titre condamnée [Ogien, 1995]. En affirmant non seulement que la rationalisation précède toutes les formes d'infraction, mais encore que c'est précisément cette "préméditation " qui rend la violation possible. Dans cette perspective, force est d'admettre que tout plongeur est en mesure d'invoquer à un moment ou à un autre de bonnes raisons pour suspendre provisoirement la validité d'une norme trop

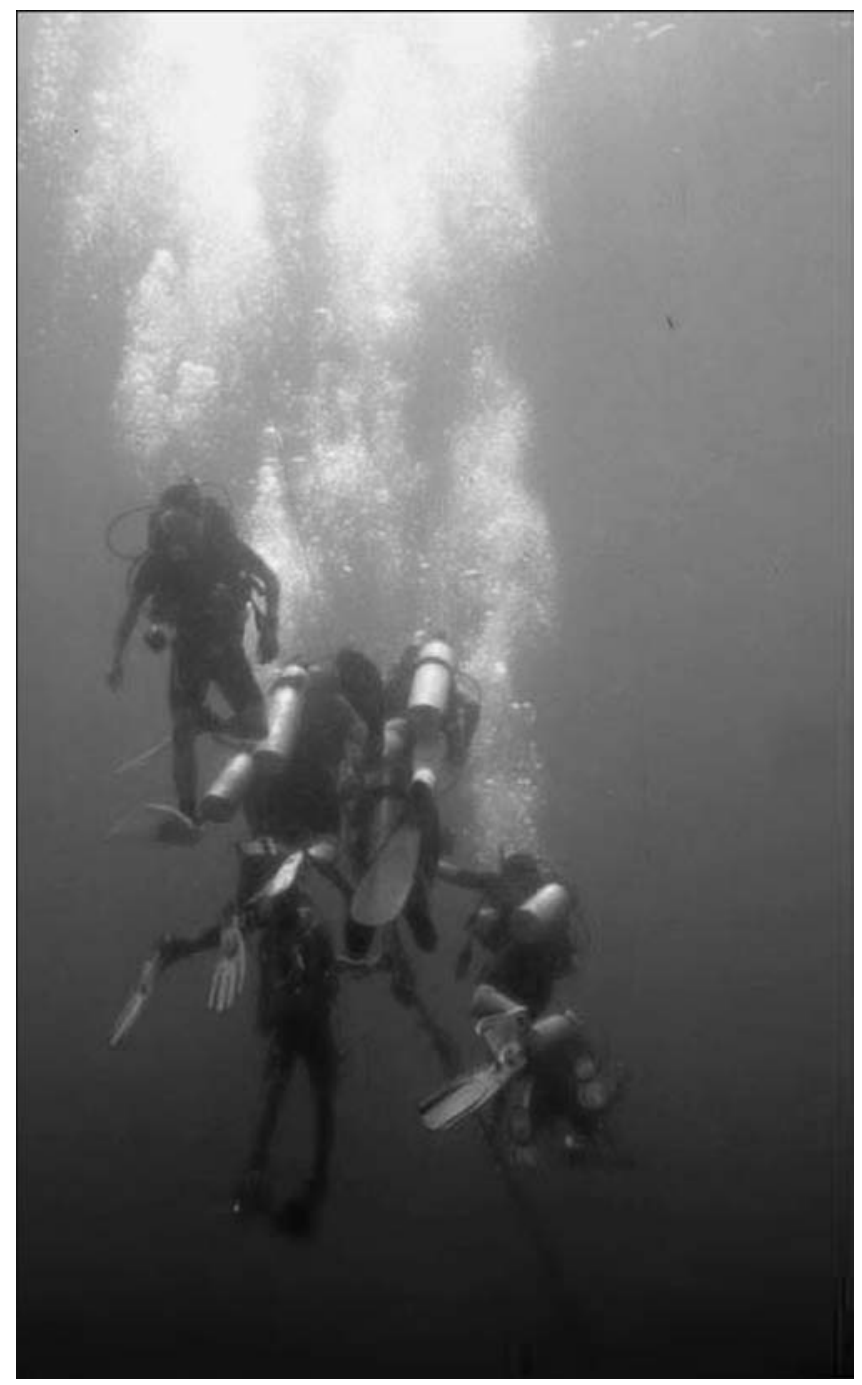

7. Plongeurs au palier des six mètres, en Corse (photo de l'auteur, 1995). 
contraignante. Ce faisant, une fois celle-ci suspendue, le ou les plongeurs se sentent comme autorisés à commettre la transgression, et ce, d'autant plus que ce type de violation mineure aura été déjà largement socialisé dans la pratique passée de l'activité. C'est ce que Gresham Sykes et David Matza, étudiant la déviance, appellent "technique de neutralisation " [Sykes et Matza, 1957]. Dès lors, en acceptant que l'acquisition des techniques de neutralisation du risque se fasse ainsi dans la pratique en même temps que celle des techniques de sécurité, et que cet apprentissage constitue une expérience partagée - en ce sens que parmi tous les risques possibles, certains vont devenir significatifs -, l'on reconnait de fait la présence du risque au cœur de la pratique. Le « ressenti » de cette présence est le produit d'interactions entre une situation donnée et l'interprétation que le plongeur est amené à faire de son expérience. La signification attribuée et la "conscience » de cette présence du risque résultent d'une intersubjectivité et relèvent d'un processus de socialisation. Les techniques de neutralisation autorisent alors les plongeurs à maintenir leur croyance dans la validité des règles légitimes de sécurité, tout en les transgressant régulièrement. Autrement dit, risque et intérêt porté à la sécurité coexistent dans la pratique normalisée de l'activité.

La plongée sous-marine, comme d'autres activités de pleine nature, cultive donc paradoxalement à la fois le goût du risque et le souci de la sécurité. Coincidentia oppositorum. On sait qu'un paradoxe est justement une contradiction qui trouve sa résolution au cœur même de l'opposition qu'elle formule. Ici, risque et sécurité poursuivent un objectif identique, de manière à la fois opposée et solidaire : celui de permettre aux plongeurs l'accès et l'exploration des fonds marins en leur procurant un plaisir et une émotion intenses, associés aux sensations nouvelles de l'immersion. Risque et sécurité promettent aux pratiquants une aventure sous-marine, la découverte, un voyage dans l'inconnu et ses mystères. Ces opposés s'étayent ainsi mutuellement afin de produire précisément cette intensité, dans des limites socialement et individuellement acceptables. Un plongeur porte ainsi en lui simultanément des tendances contradictoires dont les circonstances et les coéquipiers favoriseront ou non la manifestation. S'il y a transgression, elle sera ainsi aisément justifiée, sans pour autant remettre en question le système normatif de l'activité. Il convient de rappeler que ce même code érige également le risque (mesuré) en valeur et que, à ce titre, celui-ci contribue à distinguer les meilleurs plongeurs, ceux capables de réaliser des exploits et des performances, des autres. La prise de risque revêt aussi une indéniable dimension psychologique dont les enjeux sont l'estime de soi, la réputation personnelle, la virilité, le courage, etc. Prendre un risque n'est pas qu'une transgression et le signifiant est d'ailleurs suffisamment ambivalent pour qu'on préfere le recouvrir de la notion plus consensuelle de sécurité : on prendra ainsi "un risque en toute sécurité ".

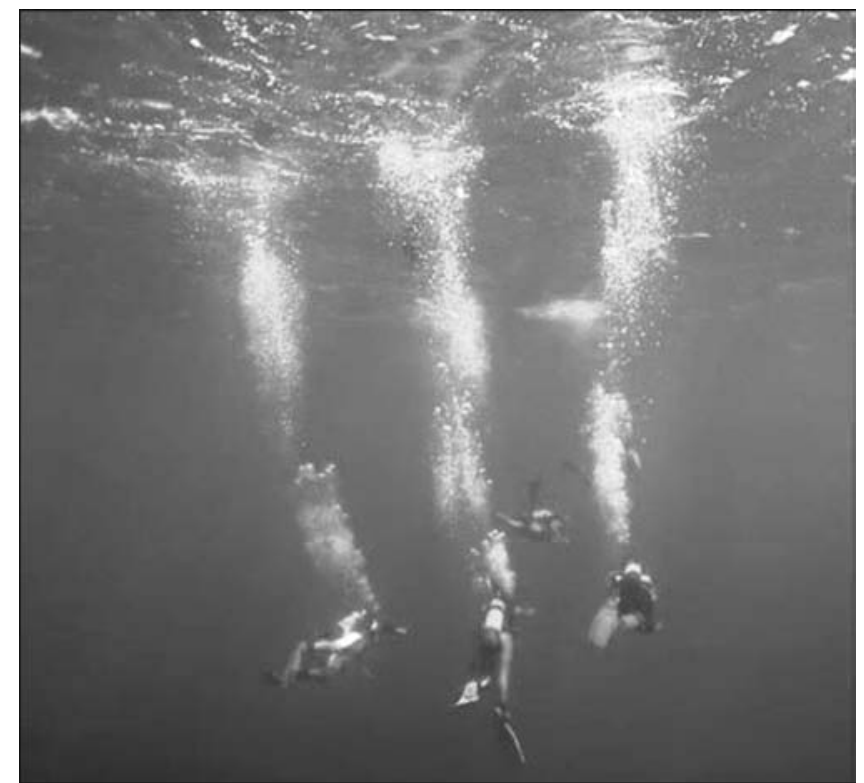

8. Début de l'immersion, après la mise à l'eau, sur la Côte d'Azur (photo de l'auteur, 1998).

Justification paradoxale, oxymore propre à une pratique qui ne peut tolérer l'existence d'un risque sans lui adjoindre son remède.

\section{- Une dénégation du risque partagée}

Comment dès lors continuer à croire que la plongée est une activité sans risque, plus sûre même dans la représentation qu'en ont les plongeurs eux-mêmes que le tennis ou la gymnastique [Collard, op. cit.] ? Comment cela est-il possible? Pour achever de répondre à cette question, on peut finalement faire deux remarques. D'abord, il faut rappeler que ces prises de risque et ce jeu avec la sécurité ne sont pas l'apanage d'une minorité de risque-tout, mais qu'ils touchent une large majorité de pratiquants. Il s'agit dans nombre de cas d'une déviance ordinaire par rapport à la pratique normale de la plongée, dont les conséquences sont généralement mineures, mais qui aboutit parfois à l'accident. Rares sont les plongeurs qui n'ont jamais connu une telle situation dans leur parcours. On se trouve alors en présence d'un risque et d'une prise de risque qui représentent une valeur commune du monde de la plongée, tout en n'ayant pas d'existence officielle. Car cela n'est évidemment jamais enseigné et toute la formation, on le conçoit, consisterait au contraire à s'en prémunir, alors même que ce risque est tributaire à la fois de la sociabilité au sein du groupe et des propriétés formelles de l'activité elle-même. Nous avons donc affaire à un risque normalisé, sans être pour autant reconnu et désigné 
comme tel. Et si cette dénégation du risque est ainsi unanimement partagée, c'est tout simplement parce que la validité du système normatif n'est à aucun moment remise en question, y compris par les transgressions. Celles-ci sont réinterprétées et justifiées dans les termes mêmes du code de prescriptions. En conséquence, les plongeurs admettent non seulement la validité des normes de comportement et des règles de sécurité dominantes, mais aussi leur justesse morale.

$\mathrm{La}$ seconde remarque concerne les plongeurs qui s'investissent davantage encore dans l'activité et pratiquent la plongée en eau profonde. Pourquoi cette minorité décide-t-elle de s'exposer ainsi plus radicalement au danger, voire de se lancer des défis ? Un premier élément de réponse réside dans le fait qu'il s'agit d'un comportement qui entre dans la même logique, mais en la poussant plus loin encore. Cet engagement plus radical permettra de mieux comprendre comment les plongeurs mettent à distance le risque. Le sentiment du risque et l'importance de sa propre exposition au danger sont indépendants de leur impact réel. Les plongeurs "profonds » distordent à leur insu la perception qu'ils ont des risques qu'ils prennent, et pour cause : cette distorsion est la condition même de leur plein engagement dans l'action. À un moment donné, il faut bien «sauter dans le vide ", y aller ou pas, et ne plus se poser de questions pour rester concentré sur la seule action. C'est ce que confirme Éric (42 ans, ingénieur, niveau IV) : "Plonger, ça doit se préparer en cinq minutes! Ça y est je saute, pour essayer de ne pas avoir cette espèce de vide!'Tu tombes à l'eau! Il faut pas que tu commences à te dire: "Attention! aujourd'hui je vais plonger, c'est profond, c'est une plongée difficile." Il faut évacuer tout ça. "Ce qu'il faut évacuer justement, c'est la conscience des risques pris et, pardelà, l'irruption de la peur. Ces plongeurs se sentent du reste peu concernés par les statistiques portant sur les accidents et la mortalité inhérents à ce type de plongée, dans la mesure où elles établissent un "risque moyen " dont ils s'excluent eux-mêmes, estimant (à juste titre) mieux plonger que la moyenne des plongeurs, et être de ce fait moins vulnérables que ces derniers. Ce mécanisme de dénégation repose sur l'affirmation de leurs qualités et compétences personnelles, sur leur capacité à contrôler la situation et sur la conviction qu'ils sauront affronter le pire. David Le Breton [2002] critique cette "illusion de contrôle " qui pousse l'individu à se croire en mesure d'échapper à la fatalité statistique et d'infléchir le destin en sa faveur. Ce mécanisme de dénégation est en parfaite cohérence avec les conceptions de ces plongeurs. Ils affirment en effet que le bon déroulement de la plongée dépend de leur capacité à s'adapter et à faire face à la situation en temps réel, à anticiper les initiatives ou les difficultés des coéquipiers et, en conséquence, à savoir quand il faut transgresser les règles ou au contraire les respecter. Cette affirmation d'un contrôle individuel ne relève cependant en rien de l'illusion, de l'optimisme absurde ou de la simple croyance en sa
" chance "; dénégation du risque et représentation de la plongée vont en effet de pair. Ces plongeurs considèrent d'ailleurs que si leur pratique intensive ne les conduit pas davantage à l'accident, c'est qu'ils ne risquent pas plus que les autres. Leur expérience régulière de la plongée renforce au contraire leurs compétences et la confiance qu'ils ont dans leur conception de l'activité. L'on comprend dès lors mieux pourquoi la plongée est conçue comme une activité sûre et sans risque.

\section{- Requalifier l'expérience du risque}

En mettant l'accent sur la pratique au détriment de la culture de la plongée, je fais l'hypothèse que la conduite des preneurs de risque et le risque lui-même dans l'activité ne pourront être convenablement appréhendés que sous l'angle de leurs caractéristiques universelles, et non sous celui de la singularité et d'une spécificité culturelle en lien avec un régime normatif donné [Matza, 1964]. Non seulement le risque n'est pas étranger à la pratique de la plongée sous-marine, mais il en est même une forme d'expression légitime (et dérangeante). Les plongeurs se saisissent du système de valeurs dominant dans la discipline et en promeuvent une partie - les valeurs indissociables de risque et de sécurité -, pour le tourner tout entier à leur avantage. Mais la valeur de risque est comme masquée et déniée au profit de celle à la fois plus respectable et plus consensuelle de sécurité. L'apprentissage et la sociabilité en plongée ne consistent pas à assimiler les seules valeurs d'une culture du risque par opposition à un système sécuritaire dominant, mais à acquérir la maitrise des techniques de neutralisation permettant au plongeur d'entretenir sa croyance dans la validité des règles de sécurité, tout en en trahissant les prescriptions. Ces procédés collectifs de requalification de la pratique et de déni de la prise de risque soulignent que la transgression des normes de sécurité est moins un acte individuel qu'une conduite déterminée par l'organisation sociale de la plongée subaquatique ${ }^{12}$. En fixant les principes auxquels les plongeurs doivent se conformer, cette dernière offre aussi les conditions qui vont conduire ceux-ci à violer ces règles, de sorte que risque et prise de risque intègrent la norme à travers un processus de renormalisation du risque en sécurité.

Le recours à la notion de technique de neutralisation permet enfin d'affirmer que la question du risque en plongée sportive n'est pas à envisager comme une sous-culture du risque, contrairement à ce que j'ai pu montrer par ailleurs pour les pêcheurs de corail [Raveneau, op. cit.]. Autrement dit, elle n'est pas à envisager comme un monde de valeurs particulières qui délimiterait une altérité, mais bien plutôt comme une forme d'action organisée autour d' "un certain rapport à la culture conventionnelle " [Ogien, op. cit.] et au code de la pratique, 
que le plongeur apprend ainsi à mettre en œuvre de manière pertinente. Toutefois, la dimension du risque et de la transgression ne recouvre pas l'ensemble de l'expérience de la plongée sous-marine, pas davantage celui du destin et des engagements sociaux du plongeur. Celui-ci est en effet traversé par bien d'autres appartenances et légitimités. Son statut comme ses différents rôles sociaux le conduisent vers des horizons variés, vers cette multiplicité des identités décrite par Erving Goffman [1961], et qui fait qu'un même individu peut être à la fois un plongeur engagé aimant le risque, un cadre modèle, un gestionnaire prudent, un père attentif, un mari impassible et un mélomane. Bref, dans la mesure où il est enserré dans un système d'obligations auquel il adhère en connaissance de cause, on peut dire que le plongeur agit toujours de façon rationnelle, quels que soient les risques qu'il prend. Fût-elle pratiquée dangereusement ou de manière atypique, son immersion s'inscrit toujours dans un monde socialement organisé.
C'est donc bien dans la compréhension interne des représentations et des pratiques du risque que réside la clef de la construction opératoire de l'identité de la plongée autour de cette question. Forgée à partir des représentations individuelles de chaque plongeur, celle-ci constitue une œuvre commune. La représentation et la dénégation du risque ne préexistent donc pas complètement au discours qui les expriment. Elles composent "une activité mimétique en tant qu'elle produit quelque chose, à savoir précisément l'agencement des faits par la mise en intrigue" [Ricœur, 1985]. C'est ce passage du "représenté " (déjà produit) à l'opératoire (se construisant) qui permet de définir l'identité du risque dans l'activité comme une dynamique pratique et non comme une donnée objective, et in fine de comprendre pourquoi les plongeurs regardent leur activité comme sûre dans la poursuite d'un but qui ne l'est pourtant pas.

\section{Notes}

1. Ce travail s'appuie sur une enquête ethnographique commencée en 1992 avec l'étude des pêcheurs de corail en Méditerranée. À partir de 1999, j'ai entamé des enquêtes consacrées à la plongée sous-marine elle-même, dans ses modalités sportives [Raveneau, 2004]. Ces travaux m'ont conduit à faire de nombreuses observations participantes (en mer comme à terre) et à m'impliquer dans la vie de clubs associatifs ou commerciaux en France comme à l'étranger (en particulier en mer Rouge).

2. Le risque se caractérise par l'incertitude relative à une situation donnée et se présente comme la mesure d'un danger potentiel dont l'accident est la matérialisation. Il doit être distingué de la prise de risque, qui souligne l'engagement volontaire du sujet dans une entreprise à l'issue incertaine.

3. Le détendeur, rattaché à la bouteille d'air sous pression, permet de fournir sous l'eau de l'air à la demande aussi sûrement qu'en surface.

4. L'air respiré sous pression occasionne un certain nombre de troubles psychiques et moteurs désignés par le terme d'ivresse des profondeurs, ou narcose. Les troubles provoqués par la respiration d'air sous pression sont caractérisés par une baisse du niveau de vigilance.

5. Luc Collard remarque, à partir de l'étude statistique des accidents de plongée recensés par un cabinet d'assurance toulousain pour la saison 1990-1991, que "si le taux d'écueil est faible (6 personnes pour 1000 sont touchées), près d'un accident sur deux est fatal et conduira à la mort si le participant n'est pas secouru sur-le-champ " [1998: 146]. Par ailleurs, les statistiques de la Fédération française d'études et de sports sousmarins (FFESSM) relèvent pour les années incluses entre 2000 et 2004 un écart compris entre 326 et 365 accidents par an, pour environ 160000 licenciés; ce qui donne un peu plus de 2 accidents pour 1000 pratiquants par an (écart compris entre 2,09 et 2,28).

6. Plongée subaquatique, considéré par beaucoup comme «la bible du plongeur ", dont la première édition date de 1972 et la dernière de 1997, a contribué à former plus de 300000 plongeurs.

7. Il arrive souvent que plusieurs bateaux se trouvent simultanément sur un même site de plongée, mais avec des moments d'arrivée et de départ plus ou moins différents.

8. Le défi ordinaire engendre des transgressions dangereuses: il faut par exemple prouver sa valeur en réussissant telle prouesse (course à la profondeur) ou en "maîtrisant " tel excès (boire de l'alcool).

9. Comme les sourds-muets, les plongeurs en immersion communiquent à l'aide de signes bien précis.

10. Il ne s'agit pas de conditions expérimentales de laboratoire, où l'on peut reproduire à l'identique les paramètres de l'expérience. Bien au contraire; les conditions de pratique de la plongée sous-marine exposent à des conditions extérieures sans cesse changeantes (courant, température de l'eau, visibilité, relief, etc.) et à une condition physique personnelle du pratiquant qui l'est tout autant.

11. Le terme utilisé couramment est "plongeur autonome".

12. On sait que l'appartenance d'individus à un groupe oriente leurs choix et constitue un biais culturel dans l'appréciation et le traitement des dangers [Douglas et Wildavsky, 1983].

\section{Références bibliographiques}

Assailly Jean-Pascal, 2001, "Sur la route, les conduites à risque ", Revue Toxibase, 2 : 1-14.

Bonnet Agnès, Jean-Louis Pedinielli, Fabien Romain, Gilles ROUAN, 2003, "Bien-être subjectif et régulation émotionnelle dans les conduites de risques. Le cas de la plongée sous-marine", L'Encéphale, 29, 6 : 488-495.
Collard Luc, 1998, Sports, enjeux et accidents, Paris, PUF.

Douglas Mary et Aaron Wildavsky, 1983, Risk and Culture, an Essay on the Selection of Technological and Environmental Dangers, Berkeley, University of California Press.

Goffman Erving, 1961, Encounters. Two Studies in Sociology of Interaction, Indianapolis, The Bobbs-Merrill Co.

Le Breton David, 1991, Passions du risque, Paris, Métailié. 
- 2002, Conduites à risque. Des jeux de mort au jeu de vivre, Paris, PUF.

Matza David, 1964, Delinquency and Drift, New York, Wiley. Molle Philippe, Pierre Rey, 1991 (1972), La plongée subaquatique, Paris, Amphora.

OgIen Albert, 1995, Sociologie de la déviance, Paris, Armand Colin.

Peretti-Watel Patrick, 2000, Sociologie du risque, Paris, Armand Colin.

POCIELlo Christian, 1995, Les cultures sportives, Paris, PUF.
RaVEneau Gilles, 1999, "Deux formes d'engagement sousmarin. Une comparaison des pêcheurs de corail corses et sardes ", Études corses, 49 : 29-53.

- 2004, «Les transformations contemporaines de la plongée sous-marine en France ", in Société de sociologie du sport de langue française, Sport en société, dispositions et pratiques sportives, Paris, L'Harmattan : 129-142.

Ricceur Paul, 1985, Temps et récit, Paris, Le Seuil.

SYKES Gresham et David MATZA, 1957, «Techniques of neutralization. A theory of delinquency ", American Sociological Review, $22: 664-670$.

\section{ABSTRACT}

Diving : between neutralization of risk and affirmation of safety

Diving in a natural medium modifies the equilibrium and perception of the body. Divers - often convinced to practice a safe activity - expose themselves to potentially mortal risks. On the basis of an ethnographic survey the author shows that this sport leaves room for an infringement of norms where risk is progressively neutralized. This implies that divers master the neutralization techniques that enable them paradoxically to maintain a normative system and to violate it at the same time. Infringement of safety norms thus appears to be an individual act rather than a behaviour determined by the divers' social organization.

Keywords : Diving. Risk taking. Norm. Infringement. Neutralization.

\section{ZUSAMMENFASSUNG}

Der Tauchsport : zwischen der Neutralisierung des Risikos und der Behauptung der Sicherheit

Der Tauchsport in einem natürlichen Medium verändert das Equilibrium und die Wahrnehmung des Körpers. Die Taucher - die oft davon überzeugt sind, dass sie einen risikolosen Sport treiben - setzen sịch potentiell tödlichen Risiken aus. Auf der Basis eiener ethnographischen Studie zeigt der Autor, dass dieser Sport Platz für eine Übertretung der Normen lässt, wo das Risiko allmählich neutralisiert wird. Dies setzt voraus, dass die Taucher die Neutralisationstechniken beherrschen, die sie paradoxerweise ermöglichen, ein normatives System zu erhalten und zur gleicher Zeit zu verletzen. Es zeigt sich, dass die Übertretung der Sicherheitsnormen eher eine individuelle Handlung als ein von der sozialen Organisation der Taucher bedingtes Verhalten ist.

Stichwörter: Tauchsport. Risikonahme. Norm. Übertretung. Neutralisation. 